\title{
Synthesis, growth, optical and thermal properties of a new organic crystal semicarbazone of p-anisaldehyde (SPAS)
}

\author{
S. Janarthanan ${ }^{1}$, Y.C. Rajan ${ }^{2}$, P.R. Umarani ${ }^{1}$, D. Jayaraman ${ }^{1}$, D. Premanand ${ }^{3}$ and S. Pandi ${ }^{*}$ \\ ${ }^{1}$ Department of Physics, Presidency College, Chennai-600 005, Tamilnadu, India \\ ${ }^{2}$ Department of Materials Science and Engineering, National Chiauo Tung University, Taiwan. \\ ${ }^{3}$ Department of Physics, St. Xavier's College, Polyamkottai, Tirnelveli, Tamilnadu, India. \\ *drspandi@gmail.com
}

\begin{abstract}
The semicarbazone of p-anisaldehyde (SPAS) is a novel organic nonlinear optical material. The SPAS has been synthesized and the solubility studies were carried out in the temperature range $30-60{ }^{\circ} \mathrm{C}$. Single crystals of SPAS have been grown following the slow evaporation technique and the unit cell parameters have been evaluated by single crystal X-ray diffraction technique. The UV-Visible, FT-IR and ${ }^{1}$ HNMR spectra have been recorded to characterize the grown crystal. The thermogravimetric and differential thermal analysis of the grown crystal revealed that there is no phase transformation in SPAS, and its thermal stability was found to be good. The dielectric measurement was made as a function of frequency $(50 \mathrm{~Hz}-5 \mathrm{MHz})$. The second harmonic generation in the sample was confirmed by the Kurtz powder technique.
\end{abstract}

Keywords: crystal growth, XRD, solution growth, thermal studies, dielectric studies.

\section{Introduction}

In recent years, the study of the design and synthesis of molecular materials with larger (hyper) polarizabilities has been a hot research topic (Elmali $A$ et al., 2005; Karakas A, Elmali A et al., 2006; Karakas A, Unver H et al., 2006). Materials showing high optical nonlinearity have potential applications in signal transmission, data storage, optical switching, etc. Due to potential applications in various photonic technologies, the NLO properties of organic molecular materials have been the object of intense research (Kanis D R et al., 1994). Using organic molecules for the nonlinear media has several advantages, such as low cost, low dielectric constant and great diversity of possible organic structures (Keinan $\mathrm{S}$ et al., 2004). Devices for applications in optical communications, optical processors, optical switches, wavelength filters and modulators have been created using the NLO response properties of organic systems. A number of organic materials showing considerable NLO effects have been identified and synthesized. However, only a few of them could be crystallized and investigated for second order NLO applications. The search for new NLO materials has been increasing as a result of growing demand for such materials. The organic molecular materials have emerged as a new class of promising nonlinear optical materials because of their superior qualities over inorganic systems. Organic compounds with asymmetric charge distribution n-electron system show large nonlinear optical responses, which have been attributed to the presence of electron rich donor and deficient acceptor substituents in the system (Chemla D S \& Zyss J 1988; Prasad P N \& Williams D J 1991; Frazier C C et al., 1987). Para anisaldehyde is chemically known as 4-methoxy benzaldehyde and used in perfume and pharmaceutical industries. Basically, semicarbazone family crystals have been found to possess nonlinear optical behaviour (Vijayan N et al., 2001; Ramesh Babu R et al., 2002). In this paper, the synthesis, growth and optical and thermal properties of a new organic crystal namely semicarbazone of $p$-anisaldehyde (SPAS) by slow evaporation solution growth technique using dimethyl formamide (DMF) as a solvent is reported. Molecular formula for semicarbazone of $p$-anisaldehyde is $\mathrm{C}_{9} \mathrm{H}_{11} \mathrm{~N}_{3} \mathrm{O}_{2}$.

The grown SPAS single crystal was confirmed by $X$ ray diffraction $(X R D)$. The functional groups and the mode of vibrations were identified by FT-IR and ${ }^{1} \mathrm{HNMR}$ spectral analysis. The optical, thermal (TGA/DTA) and dielectric properties were also investigated. Second harmonic generation (SHG) behavior of the SPAS crystal was tested by Nd: YAG laser.

\section{Materials and methods}

\section{Synthesis, Solubility and Crystal growth}

SPAS were synthesized from the starting materials namely, semicarbazide hydrochloride (S) and panisaldehyde (PAS) using sodium acetate as a catalyst in the mole ratio 1.0:1.5:0.5 respectively. The semicarbazide hydrochloride and sodium acetate were dissolved in double distilled water and p-anisaldehyde was dissolved in ethanol. Then, these two solutions were mixed. The prepared mixture was found to be turbid and ethanol was added and stirred well by using a motorized magnetic stirrer till a clear solution was obtained. The solution was taken in a covered container for controlled evaporation and kept in a constant temperature bath at 35 C. After 3 days good transparent needle shaped single crystals of SPAS were obtained. The obtained crystals were further purified by repeated recrystallisation process using ethanol as a solvent for three times. The
Janarthanan et al. Indian J.Sci.Technol. 
purified product was dissolved in the solvent dimethyl formamide (DMF) as solvent. It is noteworthy to mention here that using DMF as a solvent gives good quality crystals. The chemical reaction is shown in scheme 1.

\section{$X$-ray diffraction analysis}

A single crystal $X$-ray diffraction analysis was carried out by an ENRAF NONIUS CAD-4 X-ray diffractometer with $\operatorname{MoK}_{\alpha}(\lambda=0.1770 \AA)$ radiation to identify the structure and to determine the lattice parameter values. The powder X-ray diffraction pattern was recorded using $\mathrm{RICH}$ SEIFERT powder X-ray diffractometer with $\mathrm{Cu} \mathrm{K}_{\alpha}(\lambda=1.5428 \AA)$ radiation to verify the correctness of lattice parameter values. The powder X-ray semicarbazone of $\mathrm{p}$-anisaldehyde

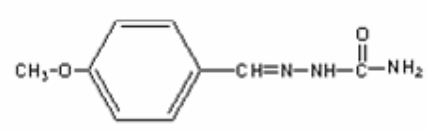

diffraction studies

\section{Scheme 1. Synthesis of SPAS}

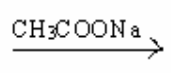

p-anisaldehyde semicarbazide hydrochloride
Solubility factor is an important parameter for crystal growth. The solubility of SPAS was analyzed using DMF as a solvent in the temperature range $30-50{ }^{\circ} \mathrm{C}$ (Fig.1).

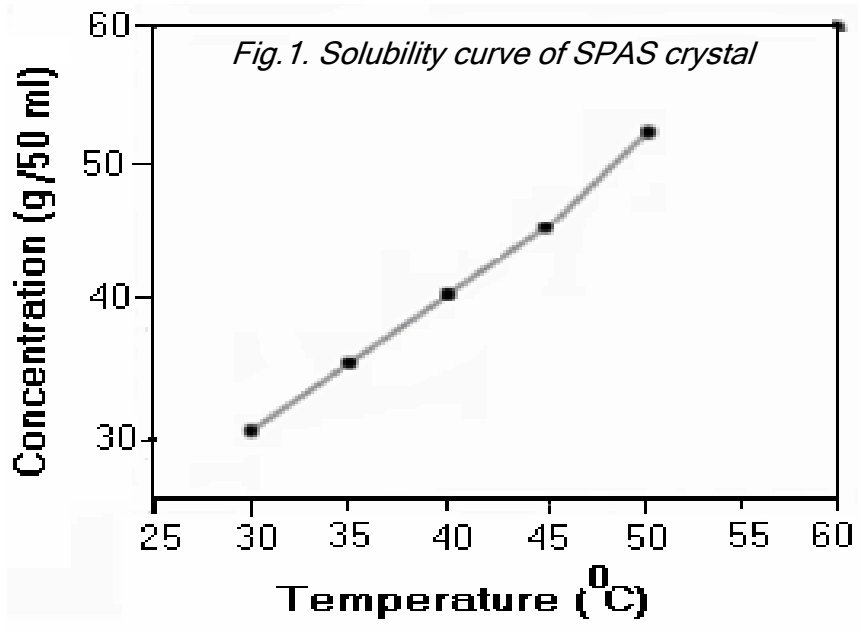

A thermostatically controlled constant temperature bath (CTB) with an accuracy of $\pm 0.01^{\circ} \mathrm{C}$ was used for this study. Solution of SPAS was prepared from the purified material and the optimally closed solution was housed in $\mathrm{CTB}$ at $35^{\circ} \mathrm{C}$ for slow evaporation. After one month, clear prismatic crystals of SPAS were harvested (Fig.2).

\section{Fig.2. Grown SPAS crystals}

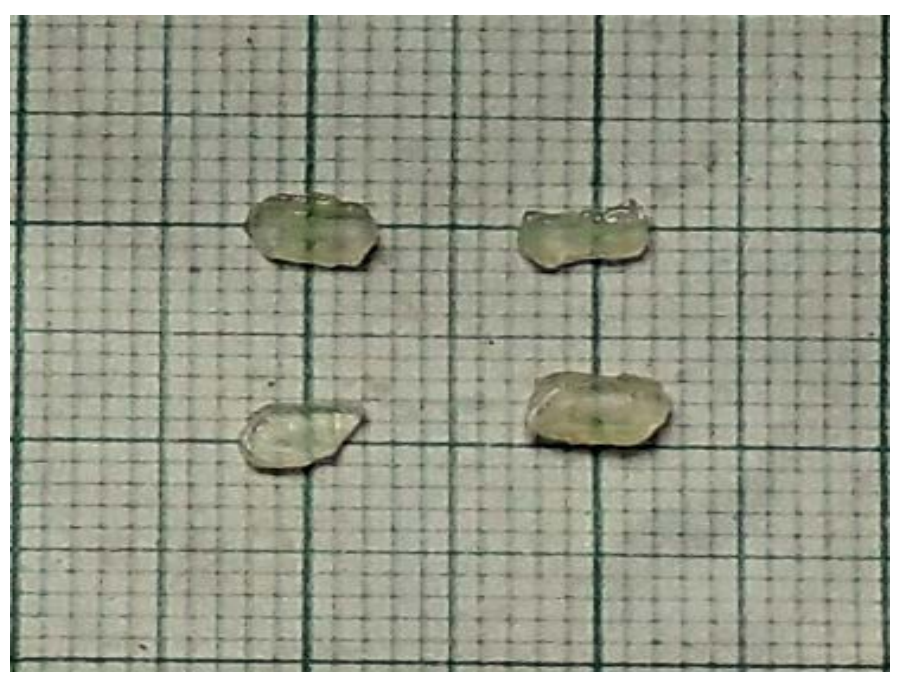

Fig.3. Powder X-ray diffraction pattern of SPAS crystal

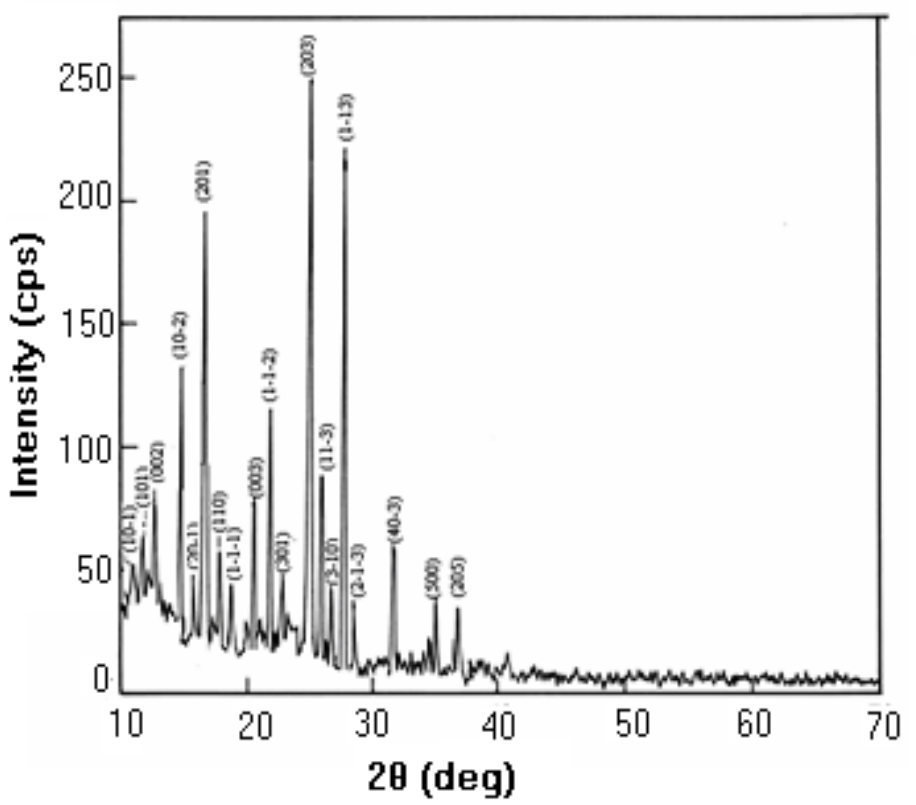

the grown crystal (Fig.3).

The experimental values were found to agree well with the calculated $\mathrm{d}$ values. The unit cell dimensions determined from single crystal X-ray diffraction analysis are presented in Table.1.

Table. 1, Crystallographic data of SPAS crystal

\begin{tabular}{|c|c|c|c|c|}
\hline $\begin{array}{c}\text { Empirical } \\
\text { formula }\end{array}$ & $\mathbf{a}(\AA)$ & $\mathbf{b}(\AA)$ & $\mathbf{c}(\AA)$ & $\begin{array}{c}\text { Crystal } \\
\text { system }\end{array}$ \\
\hline $\mathrm{C}_{9} \mathrm{H}_{11} \mathrm{~N}_{3} \mathrm{O}_{2}$ & 13.78 & 5.42 & 12.83 & \multirow{2}{*}{ Monoclinic } \\
\cline { 1 - 4 } Volume $(\AA)^{3}$ & $\boldsymbol{\alpha}$ & $\boldsymbol{\beta}$ & $\gamma$ & \\
\hline 951.64 & $90^{\circ}$ & $97.65^{\circ}$ & $90^{\circ}$ & \\
\hline
\end{tabular}

\section{Fourier transform infrared (FT-IR) spectral analysis}

The FT-IR analysis was carried out on the grown semicarbazone of $\mathrm{p}$-anisaldehyde single crystal in the region 4000 to $400 \mathrm{~cm}^{-1}$ using BRUKER IFS 66V FT-IR spectrometer to identify the functional groups present in the molecular structure of the synthesized compound (Fig.4). In order to absorb infrared radiation a molecule must undergo a net change in dipole moment as a consequence of its vibrational motion. Below $1400 \mathrm{~cm}^{-1}$
Research article

CIndian Society for Education and Environment (iSee)
Janarthanan et al. Indian J.Sci.Technol. 
Fig.4. FT-IR spectrum of SPAS crystal

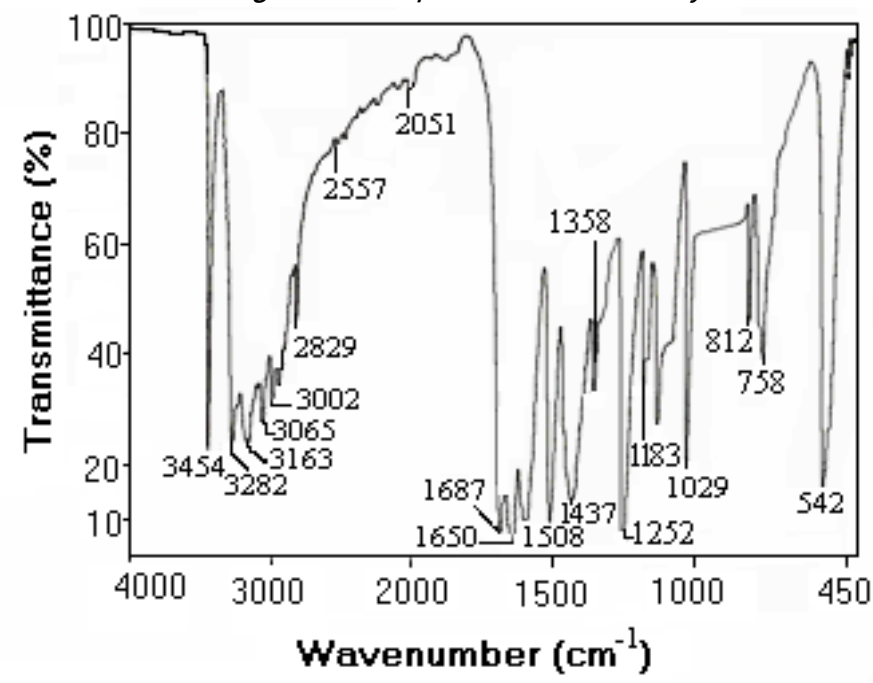

the region is called finger print region. There was an intense sharp peak at $3454 \mathrm{~cm}^{-1}$ assigned to the hydrogen bonded $\mathrm{N}-\mathrm{H}$ stretching. The broad peak obtained between 3282 and $2829 \mathrm{~cm}^{-1}$ was due to overlapping of peaks of hydrogen bonded $\mathrm{N}-\mathrm{H}$ and aromatic $\mathrm{C}-\mathrm{H}$ stretching modes. The considerable broadening revealed that the peaks of the molecules in the crystal were largely through intermolecular hydrogen bonding of these $\mathrm{N}-\mathrm{H}$ groups. The symmetric and asymmetric $\mathrm{C}-\mathrm{H}$ stretching modes of $-\mathrm{CH}_{3}$ group appeared as a shoulder just below $3000 \mathrm{~cm}^{-1}$ in the broad envelope. The $\mathrm{C}=\mathrm{O}$ stretching of semicarbozide moiety was observed at $1687 \mathrm{~cm}^{-1}$ and the peak at $1650 \mathrm{~cm}^{-1}$ was assigned to $\mathrm{C}=\mathrm{N}$ stretching vibration. The $\mathrm{N}-\mathrm{H}$ bending vibration gave a peak at $1508 \mathrm{~cm}^{-1}$. The $-\mathrm{CH}_{3}$ bending mode was positioned at $1437 \mathrm{~cm}^{-1}$. The bands obtained between 1183 and $1252 \mathrm{~cm}^{-1}$ were due to $-\mathrm{OCH}_{3}$ groups.

\section{Proton nuclear magnetic resonance ( $H N M R)$ spectral analysis}

Identification of compounds is an important task and is very much accomplished by technique like NMR spectral analysis (Bruice $P$ Y, 2002). The ${ }^{1}$ HNMR spectrum of SPAS was recorded using JOEL GSX 500 instrument to get complete idea of the carbon-hydrogen framework of the compound (Fig.5).

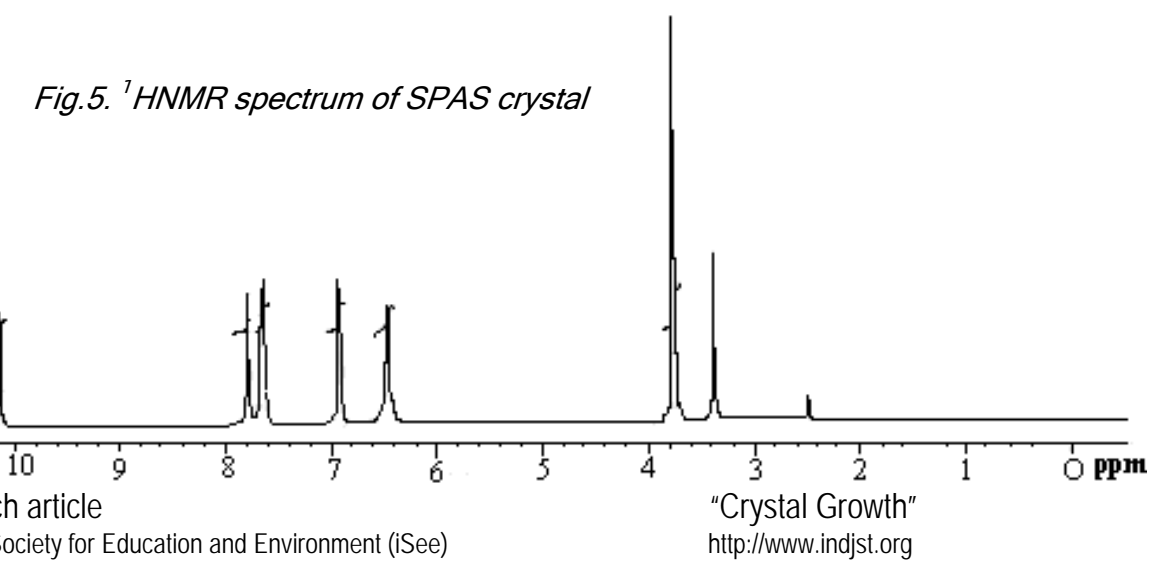
azomethine moiety.

\section{Thermal analysis}

Vol. 3 No. 8 (Aug 2010)

ISSN: 0974- 6846

The SPAS in its powder form was dissolved in DMSO- $d_{6}$ containing TMS as an internal standard. A sharp singlet at $3.73 \mathrm{ppm}$ was due to the presence of methoxy group. $\mathrm{NH}_{2}$ and $\mathrm{NH}$ groups of semicarbazone moieties were observed at 6.42 and $6.91 \mathrm{ppm}$, respectively. A doublet observed at $7.62 \mathrm{ppm}$ was due to aromatic proton. The remaining four aromatic protons were observed in the region between 7.01 and $8.00 \mathrm{ppm}$ as a multiplet. The signal at $10.14 \mathrm{ppm}$ was due to $-\mathrm{CH}$ proton of the

\section{UV-Visible spectral analysis}

The optical transmission spectrum for the wavelengths between 200 and $1000 \mathrm{~nm}$ was recorded using the instrument Varian CARY 5E UV-Vis-NIR spectrophotometer with an optically polished SPAS single crystal of thickness $2 \mathrm{~mm}$ (Fig.6).

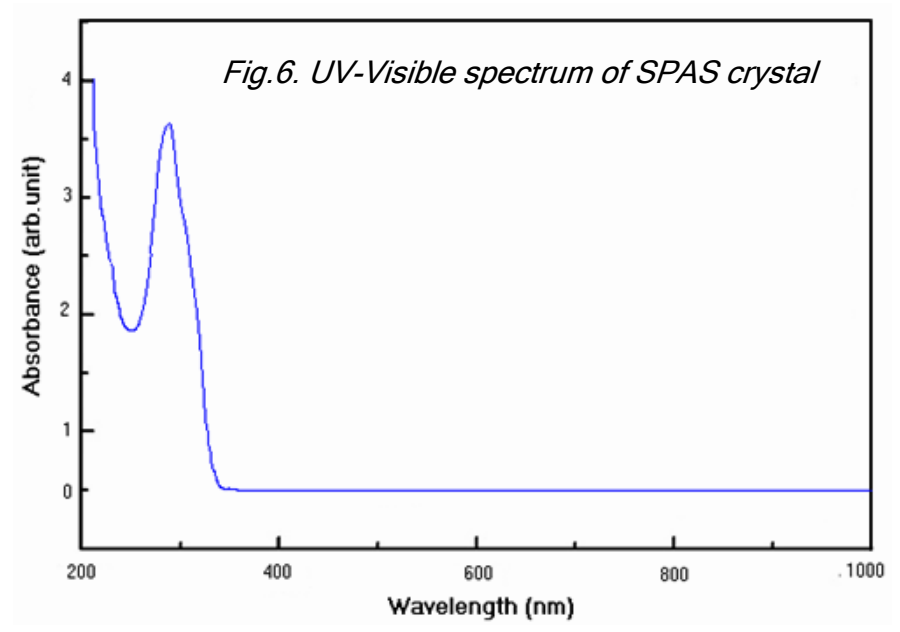

The UV spectral studies were very important for any NLO materials because NLO materials must have a wide transparency window for optical applications. The transmittance was found to be maximum in the entire UVvisible region and the UV cut-off wavelength of SPAS was below $300 \mathrm{~nm}$. There was no absorption of light in the UV-visible range of the electromagnetic spectrum and it can be used as a potential material for SHG or other applications in the blue and violet regions.

In order to know the thermal behavior of the material, the sample was subjected to thermogravimetric analysis. The thermogravimetric analysis (TGA) and differential thermal analysis (DTA) were carried out using NETZSCH STA $409 \mathrm{C} / \mathrm{CD}$ thermal analyzer at a heating rate of $10{ }^{\circ} \mathrm{C}$ $\mathrm{min}^{-1}$ in the nitrogen atmosphere (Fig.7). No weight loss was observed below $200{ }^{\circ} \mathrm{C}$. This indicates that there was no inclusion of water in the crystal 
lattice. The TG spectrum showed a major weight loss from $305.2{ }^{\circ} \mathrm{C}$ to $372{ }^{\circ} \mathrm{C}$. The nature of weight loss

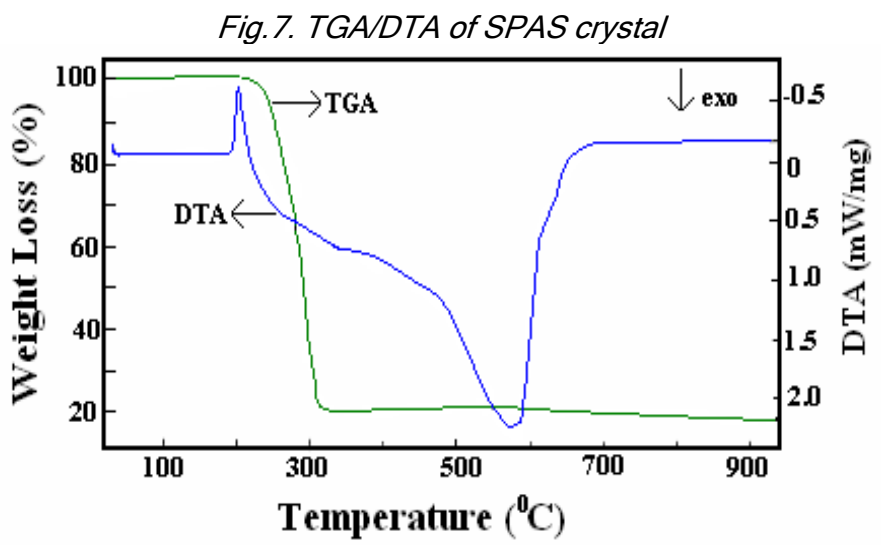

indicated the decomposition point of the sample. However, below this temperature no weight loss was observed. The peak observed around $300{ }^{\circ} \mathrm{C}$ corresponds to the decomposition temperature of the sample. The DTA trace showed exothermic peak around $200{ }^{\circ} \mathrm{C}$, which was assigned to intense weight loss of the material. Hence, this compound was found to undergo decomposition before melting. There was no phase transition before the decomposition point of $200{ }^{\circ} \mathrm{C}$. Hence, this material was found to have a very good thermal stability up to $200{ }^{\circ} \mathrm{C}$.

\section{Dielectric studies}

The Dielectric properties normally correlate with electro-optic property of crystals (Boomadevi $S$ \& Dhanasekaran $R$ 2004). Hence, the grown SPAS single crystal was subjected to dielectric studies using a HIOKI 3532 LCR instrument. The cut and polished single crystal of SPAS was used for dielectric studies. The surface of

Fig.8. Variation of dielectric constant of SPAS crystal as a function of frequency at various temperatures

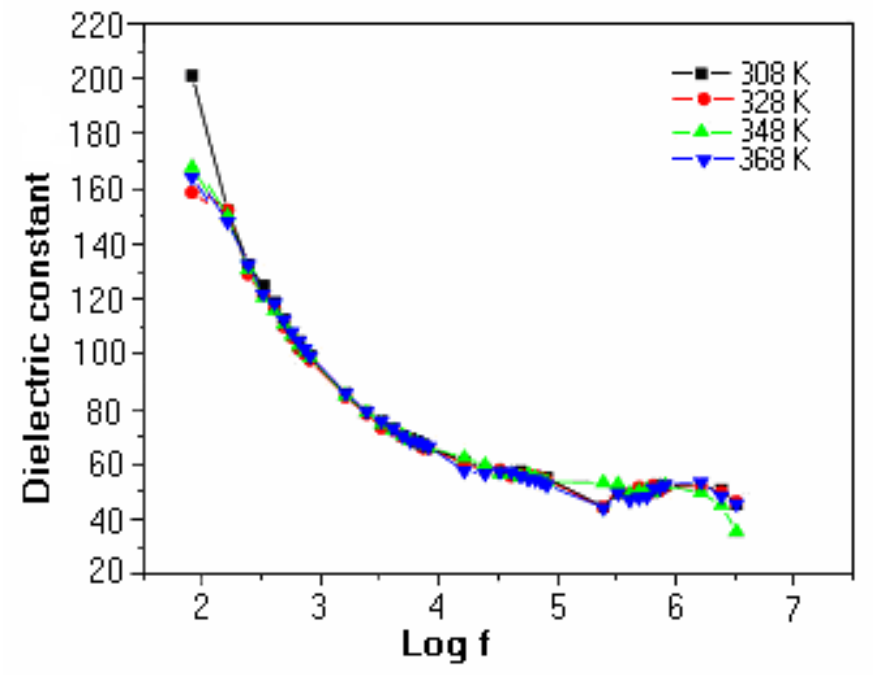

Vol. 3 No. 8 (Aug 2010)

ISSN: 0974- 6846 the sample was electroded with silver paste for electrical contact. The experiment was carried out for the frequencies from $50 \mathrm{~Hz}$ to $5 \mathrm{MHz}$ at the temperatures $308 \mathrm{~K}, 328 \mathrm{~K}, 348 \mathrm{~K}$ and $368 \mathrm{~K}$. In general, the dielectric response would be good in the lower frequency region for the organic crystals. Hence, the experiments were carried out in the lower frequency region only. The dielectric constant $\varepsilon_{r}$ (Fig.8) was calculated using the relation, $\varepsilon_{r}=C d / \varepsilon_{0} A$ where, $C$ is the capacitance, $d$ is the thickness of the crystal, $\varepsilon_{0}$ is the vacuum dielectric constant and A is the area of the crystal. The dielectric loss $\varepsilon$ " (Fig.9) was calculated using the relation $\varepsilon^{\prime \prime}=\varepsilon_{\mathrm{r}} D$ where, $D$ is the dissipation factor. From these graphs, it was found that the values of dielectric constant and dielectric loss decrease with increase in frequency. The very high values of $\varepsilon_{\mathrm{r}}$ at low frequencies might be due to the presence of all the four polarizations namely, space charge, orientational, ionic and electronic polarizations and its low value at high frequencies might be due to the loss of significance of these polarizations gradually (Prasad N V et al., 1996). The low value of dielectric loss at high frequencies revealed the high optical quality of the crystal with lesser defects, which was a desirable property for NLO applications.

\section{NLO property studies}

Current interest in quantum electronics is focused on finding materials which have suitable non-linear optical properties for use as the active media in efficient second harmonic generators, tunable parametric oscillators and broadband electro-optic modulators. The grown crystal of SPAS was subjected to Kurtz Second Harmonic Generation (SHG) test for the non-linear optical (NLO) property (Kurtz S K \& Perry T T 1968). The crystal was illuminated by the laser beam with first harmonic output of $1064 \mathrm{~nm}$ and also with a pulse width of $8 \mathrm{~ns}$ using $\mathrm{Nd}$ :

Fig.9. Variation of dielectric loss of SPAS crystal as a function of frequency at various temperatures

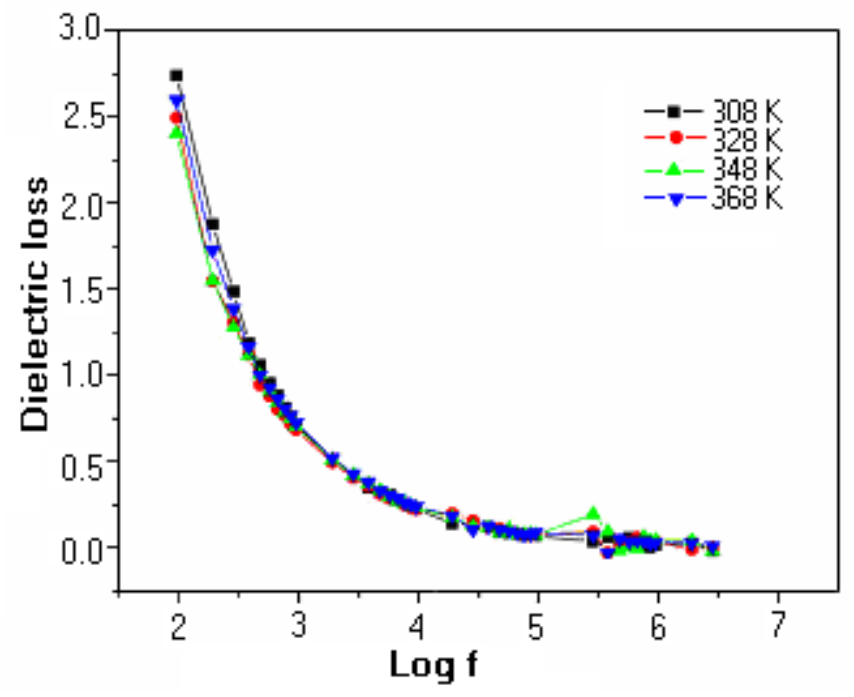

Research article CIndian Society for Education and Environment (iSee)
"Crystal Growth" http://www.indjst.org
Janarthanan et al. Indian J.Sci.Technol. 
YAG Q-switched laser source. The generation of second harmonic wave was confirmed by the emission of green radiation from the sample.

\section{Conclusions}

Good quality single crystals of SPAS were grown successfully for the first time by the slow evaporation solution growth method from dimethyl formamide (DMF). The crystal system was identified by the single XRD studies. From the powder X-ray diffraction pattern, the lattice parameters were calculated and the monoclinic structure was confirmed. From the FT-IR and proton NMR spectral assignments, the presence of functional groups in the title compound has been confirmed. The thermal behavior of the SPAS crystal was analyzed by TGA and DTA studies and the thermal stability of the material was determined. Optical assessment shows that it has a large transmission window and it might be used for frequency doubling and other NLO applications. Also, temperature dependence dielectric studies revealed increase in dielectric constant with increase in temperature. The low dielectric loss enumerated that the grown crystal has only minimum defects. The SHG of the sample was confirmed by the Kurtz technique. The SHG efficiency measurements and etching studies are in progress.

\section{Acknowledgements}

It is a pleasure to acknowledge Dr. M. Vanjinathan, Asst.Professor, Department of Chemistry, D.G.Vaishnava College, Chennai and Dr. V. Manivannan, Scientist, PRIST University, Tanjour for their constant help, support and encouragement.

\section{References}

1. Boomadevi S and Dhanasekaran RD (2004) Synthesis, crystal growth and characterization of L-pyrrolidone-2-carboxylic acid (L-PCA) crystals. J. Cryst. Growth. 261,70-76.

2. Bruice PY (2002) Organic chemistry, Pearson education (Singapore) Pte. Ltd., first Indian reprint, Delhi. 534-565.

3. Chemla DS and Zyss J (1988) Non-linear optical properties of organic molecule and crystals. 1 \& 2, Academic Press, NY.

4. Elmali A, Karaka A and Unver H (2005) Non-linear optical properties of bis[( $p$-bromophenylsalicylaldiminato)chloro] iron(III) and its ligand $\mathrm{N}$-(4bromo)-salicylaldimine. Chem. Phys. 309, 251.

5. Frazier CC, Cockerham MP, Chauchard EA and Lee $\mathrm{CH}$ (1987) Second-harmonic generation in aromatic organic compounds. J. Opt. Soc. Am. B. 4, 18991903.

6. Kanis DR, Ratner MA and Marks TJ (1994) Design and construction of molecular assemblies with large second-order optical nonlinearities. Quantum chemical aspects. Chem. Rev. 94, 195-242.
Vol. 3 No. 8 (Aug 2010)

ISSN: 0974- 6846

7. Karakas $A$, Elmali $A$, Unver $H$, Kara $H$ and Yahsi $Y$ (2006a) Synthesis, structure, spectroscopic studies and ab-initio calculations on first hyperpolarizabilities of $\quad \mathrm{N}, \mathrm{N}$ '-Bis(2-hydroxy-1-naphthylmethylidene)-1methyl-1,2-diaminoethane-N,N',O,O'-copper(II). $Z$. Naturforsch. 61b, 968-974.

8. Karakas A, Unver H and Elamli A (2006b) Static and dynamic second hyperpolarizabilities of $[\mathrm{FeL}(\mathrm{MeOH}) \mathrm{Cl}]_{2} \quad(\mathrm{~L} \quad=\quad N-(5$-methylphenyl)-3methoxysalicylaldimine). J. Mol. Struct. (Theochem.). 774, 67-70.

9. Keinan S, Ratner MA and Marks TJ (2004) Selfassembled electrooptic superlattices. A theoretical study of multilayer formation and response using donor-acceptor, hydrogen-bond building blocks. Chem. Mater. 16, 1848-1854.

10.Kurtz SK and Perry TT (1968) A powder technique for the evaluation of non-linear optical materials. J. Appl. Phys. 39, 3798-3813.

11.Prasad NV, Prasad G, Bhimasankaram T, Suryanarayana S V and Kumar GS (1996) Dielectric properties of cobalt doped cadmium oxalate crystals. Bull. Mater. Sci. 19, 639-643.

12.Prasad PN and Williams DJ (1991) Introduction to non-linear optical effects in molecules and polymers, Wiley, NY. 1003-1008.

13.Ramesh Babu R, Vijayan N, Gopalakrishnan R and Ramasamy P (2002) Growth and characterization of benzaldehyde semicarbazone (BSC) single crystals. J. Cryst. Growth. 240, 545-548.

14. Vijayan N, Ramesh Babu R, Gopalakrishnan R, Dhanuskodi S and Ramasamy P (2001) Growth and characterization of organic crystals of semicarbazone of acetophenone. J. Cryst. Growth. 233, 863-867.
Janarthanan et al. Indian J.Sci.Technol. 